\title{
SYSTEM CONSIDERATIONS AND CHALLENDES IN 3D MAPPING AND MODELING USING LOW-COST UAV SYSTEMS
}

\author{
Z. Lari*, N. El-Sheimy \\ Department of Geomatics Engineering, University of Calgary, 2500 University Dr. NW, Calgary, AB, Canada T2N 1N4
}

KEY WORDS: Unmanned Aerial Vehicle (UAV), System architecture, Calibration, Integration, Processing

\begin{abstract}
:
In the last few years, low-cost UAV systems have been acknowledged as an affordable technology for geospatial data acquisition that can meet the needs of a variety of traditional and non-traditional mapping applications. In spite of its proven potential, UAV-based mapping is still lacking in terms of what is needed for it to become an acceptable mapping tool. In other words, a well-designed system architecture that considers payload restrictions as well as the specifications of the utilized direct geo-referencing component and the imaging systems in light of the required mapping accuracy and intended application is still required. Moreover, efficient data processing workflows, which are capable of delivering the mapping products with the specified quality while considering the synergistic characteristics of the sensors onboard, the wide range of potential users who might lack deep knowledge in mapping activities, and time constraints of emerging applications, are still needed to be adopted. Therefore, the introduced challenges by having low-cost imaging and georeferencing sensors onboard UAVs with limited payload capability, the necessity of efficient data processing techniques for delivering required products for intended applications, and the diversity of potential users with insufficient mapping-related expertise needs to be fully investigated and addressed by UAV-based mapping research efforts. This paper addresses these challenges and reviews system considerations, adaptive processing techniques, and quality assurance/quality control procedures for achievement of accurate mapping products from these systems.
\end{abstract}

\section{INTRODUCTION}

Recently, accurate 3D mapping and modelling of our environment have become prerequisite for different applications such as urban planning, 3D building modelling, archaeological documentation, environmental monitoring, pipeline inspection, infrastructure inventory and monitoring, landslide hazard assessment, open pit mining, and indoor mapping. The demands of these emerging applications cannot be satisfied by traditional mapping using costly and high-end data acquisition systems, due to limitations in financial and technical resources. Recent advances in hardware (low-cost high resolution digital cameras, laser scanners, and navigation systems) and software development have made it possible to conduct accurate $3 \mathrm{D}$ mapping without using costly mapping-grade data acquisition systems. Moreover, remarkable developments in Mobile Mapping Technology (MMT) have made accurate 3D mapping more feasible whenever and wherever required (El-Sheimy, 2005; Tao and Li, 2007). In spite of the significant impact of MMT on human-operated terrestrial and airborne platforms in terms of providing a high-quality mapping products, the initial investment and mobilization cost, and the required technical expertise of end users are preventing their widespread adoption by potential individuals who might benefit from such technology.

In the past few years, Unmanned Aerial Vehicles (UAVs) have been evolving as an cost-effective and flexible alternative to traditional mobile mapping platforms for providing high quality mapping products (Eisenbeiss, 2011). These platforms bridge the gap between traditional airborne and terrestrial mobile mapping systems, in terms of the extent of area to be mapped as well as accessibility constraints (Neitzel and Klonowski, 2012).
More specifically, the main advantages of UAVs for mapping and modelling applications, can be summarized as follows: (a) they can be cost-effectively stored and deployed, which make them optimal for rapid-response mapping applications (Everaerts, 2008); (b) they can fly at lower altitude and slower speed than manned aircrafts, thus providing high quality spatial data; (c) they can perform missions and acquire data autonomously so that human operation is minimized; (d) they are highly manoeuvrable, which is ideal for low altitude flying and complex environments; (e) they can provide repetitive mapping at higher frequency with minimal cost; and (f) they can operate in adverse weather and dangerous environments.

In spite of its proven potential, the research related to UAVbased mapping is opposed to several challenges for deriving accurate products. For example, a well-designed system architecture that considers payload restrictions as well as specifications of the utilized geo-referencing and imaging sensors while considering the required mapping accuracy and intended application is still lacking. Moreover, the development of a data collection mission planning and a data processing workflow, which are capable for providing data and delivering the mapping products with the specified quality while considering the synergic characteristics of the sensors onboard and wide range of potential users, is still missing. In other words, the introduced challenges by having low-cost imaging (cameras and laser scanners) and geo-referencing sensors onboard UAVs with limited payload capability, ensuring stability of the system calibration parameters, optimal mission planning, accurate integration of imaging systems and direct geo-referencing units onboard, adaptive processing of acquired data by these system, quality control of final product, and diversity of potential users with insufficient mapping-related

\footnotetext{
* Corresponding author
} 
expertise have not been fully addressed and considered by current research. Therefore, considerable research efforts are still needed to take the aforementioned challenges into account and exploit potential benefits of UAV systems for different mapping and modelling applications. This paper reviews the encountered challenges in using low-cost UAV systems as mobile mapping platforms and introduces alternatives for resolving these challenges and deriving high quality mapping products from these systems while considering the requirements of a wide-range of applications.

\section{SYSTEM CONSIDERATIONS}

Due to their flexibility and cost saving, UAV systems have evolved as an alternative to traditional mapping platforms for a wide range of applications. In these systems, the balance between payload capacity and the required accuracy for the final product is considered as the most important posed challenge. UAV payload restrictions enforce the use of lower-grade (consumer-grade) sensors which in turn will negatively affect the quality of the final product (Nex and Remondino, 2014). In this section, the challenges arising from the use of lower-quality imaging and geo-referencing sensors in low-cost UAV system will be discussed in detail.

\subsection{UAV Multi-Sensor System Architecture}

To ensure that a UAV system will be capable of providing information that satisfies the demands of a wide range of applications, the specifications and arrangement of necessary system components which are commensurate with the payload restrictions, extent of the area to be mapped, and the required accuracy should be investigated. UAVs, which are designed and utilized for mapping applications, are usually equipped with direct geo-referencing units, passive and/or active optical imaging systems (digital cameras and/or laser scanners). The direct geo-referencing unit onboard - GNSS/IMU - is employed to navigate UAV platform and determine its position and orientation during optical data acquisition. The main advantage of direct geo-referencing units is reducing or even eliminating the ground control requirement, which is quite beneficial for mapping inaccessible areas as well as reducing the overall cost of mapping procedure. In order to consider the payload restrictions, low-cost UAV systems usually include low-cost GNSS/IMU systems such as a compact GPS, a lowcost Fiber Optic Gyro (FOG) and/or Micro-Electro-Mechanical (MEMS) inertial sensors. These sensors should be carefully integrated to provide an accurate and reliable platform navigation solution as economically as possible both in terms of price and power.

Furthermore, the intended UAVs usually include a maximum of one lightweight active optical imaging unit and one or more passive optical imaging systems (low-cost digital cameras) for geo-spatial data acquisition. The utilization of multiple synchronized imaging sensors, where payload restrictions allow, is also performed to ensure extended coverage of the scanned area. The balance between the UAV payload capacity and the required accuracy for the final mapping product is one of the key challenges of UAV-based mapping. For example, UAV payload restrictions might enforce the use of lower-grade (consumer-grade) sensors which in turn will negatively affect the quality of the final product (Chiang et al., 2012). Alternatively, the use of large UAVs with heavier payloads, which can handle mapping-grade sensors, would sacrifice the cost benefit. Therefore, different research activities have been conducted in the past few years are to address the challenges arising from the use of lower-quality mapping sensors while maintaining the quality of the mapping outcome (Nagai et al., 2009; Pfeifer et al., 2012; Wallace et al., 2012; Rehak et al., 2013).

The next important consideration in system architecture design is structural integrity and optimum arrangement of different sensors relative to each other to provide the required overlap between the collected data by these sensors. The integrity of the involved sensors (hardware integrity) in UAV systems should also be frequently investigated to ensure the correctness of the information supplied by the total system (Skaloud, 2007). To help with figuring out the optimum alignment of the different sensors, a simulation environment has been developed to quantitatively evaluate the capabilities of different proposed configurations in capturing geospatial information for various applications (e.g., urban mapping, landslide-risk areas, volumetric change detection, architectural documentation, pipeline inspection, and infrastructure inventory and monitoring). This environment will be a quite helpful tool for system architects and decision makers. Figure 1 shows a view of this simulation environment.

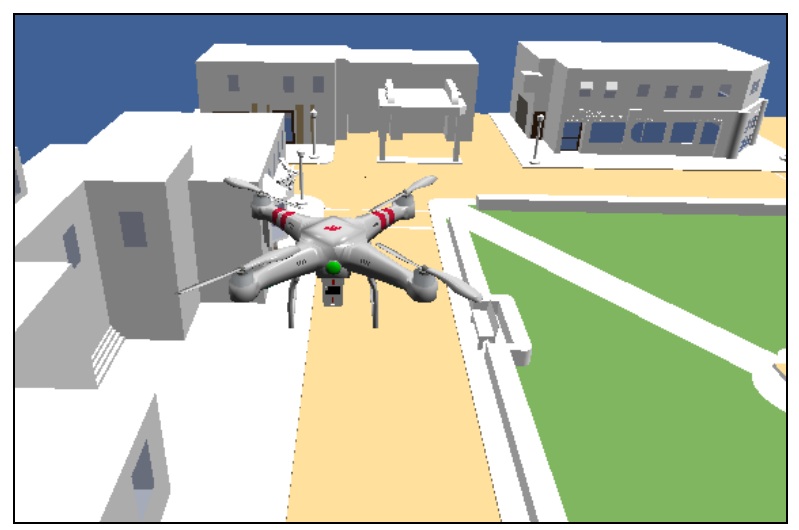

Figure 1. UAV-based mapping simulated environment

\subsection{System Calibration}

After deciding on the sensors' specifications and arrangement in a UAV system, efficient system calibration techniques should be applied to ensure achievement of the potential accuracy of these systems for different mapping applications (El-Sheimy, 1996; Pinto and Forlani, 2002; Cramer and Stallmann, 2002; Habib et al., 2010). In this case, system calibration would entail both the internal sensor characteristics (e.g., focal length and distortions of the lenses associated with the utilized digital cameras as well as systematic biases in laser scanning systems' measurements - i.e., encoder angles and laser ranges) and the external sensor characteristics (i.e., the mounting parameters spatial and rotational offsets - relating the direct georeferencing unit and the passive/active imaging systems) (Habib et al., 2011; Kersting et al., 2011). Since we are dealing with consumer-grade imaging sensors in low-cost UAV systems, a more careful system calibration will be essential to accurately determine internal and external sensor characteristics. Traditionally, the system calibration procedure was performed for passive and active imaging sensors, separately (Lin et al., 2011; Zhou et al., 2012; Kwak et al., 2013). The reliability of this calibration procedure is highly dependent on the availability of a calibration site with well-distributed ground control points and strong data acquisition geometry which are not always accessible and possible when using UAV systems for mapping in remote areas. In order to overcome these shortcomings, novel techniques have been proposed in recent 
years which focus on simultaneous incorporation of active and passive imaging units in the system calibration process (Lari et al., 2014; Habib et al., 2011). One advantage of such simultaneous procedures is that the synergistic characteristics of the data acquired using passive and active imaging systems would allow for a better calibration while relying on fewer control points. Moreover, they ensure the coalignment/registration of the passive and active sensory data which will be quite valuable for subsequent processing steps. Figures 2.a and 2.b show the impact of accurate system calibration on the quality of a reconstructed 3D surface using images acquired by a low-cost UAV system (DJI Phantom II). As seen in Figure 2.a, the quality of the reconstructed 3D surfaces using the collected images has deteriorated due to improper system calibration (inaccurate estimation of lens distortion parameters for the fish-eye camera mounted on the UAV system).

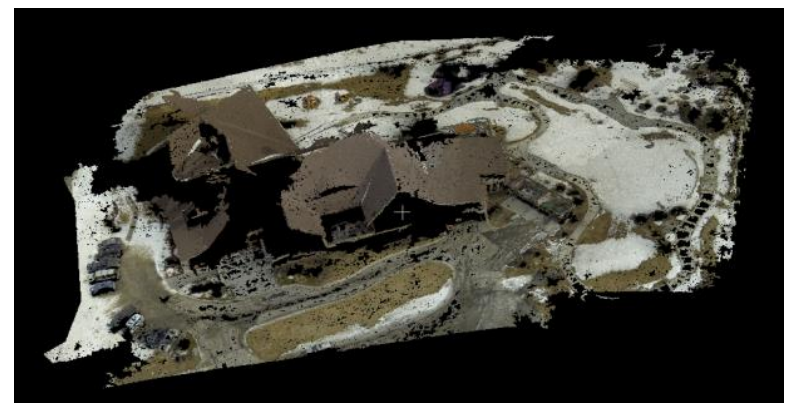

(a)

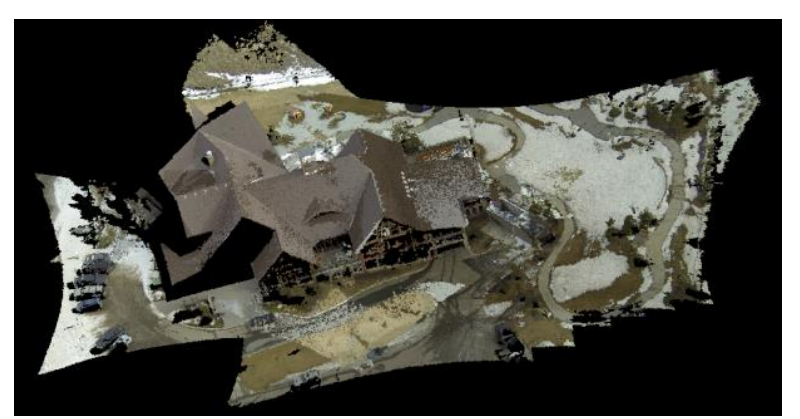

(b)

Figure 2: The impact of accurate system calibration on the quality of reconstructed 3D surface using images collected by a low-cost camera mounted on a UAV system: (a) reconstructed

3D surface without considering accurate system calibration parameters, and (b) reconstructed 3D surface while considering accurate system calibration parameters

Considering the fact that structural stability of low-cost UAV systems is not a part of their architecture design criteria, specific techniques should also be utilized to analyse the stability of the multi-sensor system calibration parameter. This stability analysis can be either performed based on statistical testing of the estimated calibration parameters from different epochs (Shortis and Beyer, 1997; Habib and Morgan, 2005; Lichti et al., 2009) or implemented based on quantitative evaluation of the impact of deviations in the temporal system parameters on the final mapping products (Habib et al., 2006; Rieke-Zapp et al., 2009; Habib et al., 2014). The advantage of the second group of stability analysis techniques is providing helpful insights for decision making regarding the suitability of the utilized sensors for intended applications. In this papers, existing approaches for system calibration and stability analysis of calibration parameters in low-cost UAV system are reviewed in detail while highlighting the their advantages and disadvantages .

\section{UAV-BASED MAPPING CHALLENGES}

In this section, the challenges in using low-cost UAV systems for geospatial data collection, processing, product delivery, and product quality control are reviewed and possible solutions for addressing these issues are addressed in detail.

\subsection{Mission planning and data acquisition}

Due to payload restrictions and limitations in architecture design, a careful mission planning for data acquisition is essential to achieve given objectives of different mapping and modelling applications (Kraus, 2007; Aber et al., 2010). A successful mission plan should take the area of interest specifications of the utilized sensors (geo-referencing and imaging), payload weight restrictions and endurance, speed, weather conditions, and intended application requirements into account. These considerations are made to determine the optimum flight path, flying altitude, collected data storage requirements, and fuel and power consumption in a UAV-based mapping project (Eisenbeiss, 2009). The aforementioned simulation environment can also be utilized for optimum flight planning while considering the sensor characteristics and the demands of the intended applications. Figure 3 shows an overview of a flight plan in a simulated environment.

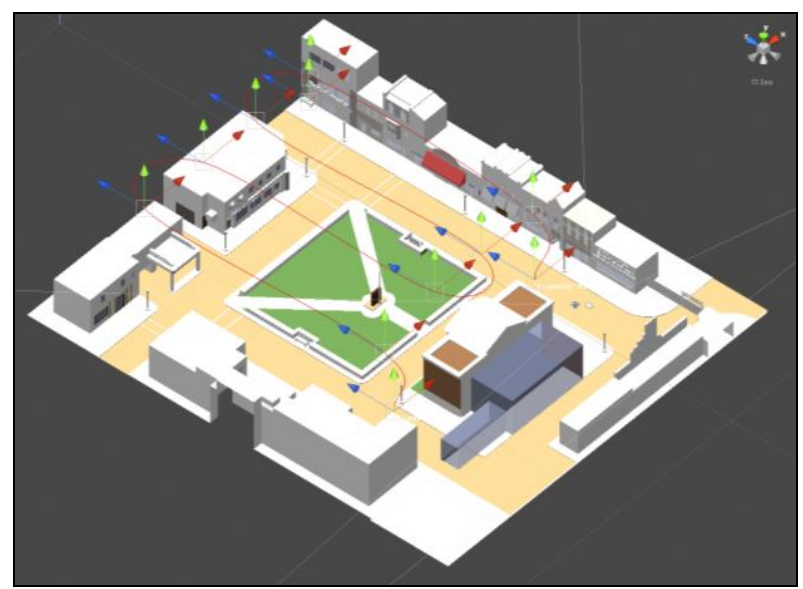

Figure 3: a simulated flight plan for 3D building modelling application

\subsection{Multi-sensor data integration}

As mentioned earlier, UAV systems have been established as low-cost multi-sensor systems and employed as alternatives to traditional mobile mapping systems. Therefore, passive/active optical imaging systems should be efficiently integrated with the direct geo-referencing sensors for enhancing the accuracy and reliability of the derived information. In this regard, the preliminary product from such systems is provided as a denselydistributed point cloud along the scanned surfaces within the field of view of the imaging sensors. The choice of point cloud as the preliminary outcome from these system is based on the fact that it is the most suitable product that could simultaneously consider the synergistic characteristics of the involved passive and active imaging systems. Furthermore, it will allow for the derivation of a wide range of mapping products to be utilized for different applications. To generate a dense point cloud from the imagery (collected by low-cost 
cameras mounted on low-cost UAV systems), automated dense matching techniques are utilized (Seitz et al., 2006; Hirschmuller, 2008; Zhu et al., 2010; Furukawa and Ponce, 2010; Gehrke et al., 2012; Furukawa and Ponce, 2010). The dense matching procedures benefit from the direct georeferencing capability and the system calibration parameters for the restriction of the search space for conjugate points in overlapping imagery (He and Habib, 2014). Moreover, the point cloud collected by laser scanning systems can be used to further restrict this search space. Accurate registration of imagery data and laser scanning data, which is implemented during the system calibration procedure, has a great impact on effective utilization of laser-based point cloud for the generation of image-based point cloud.

In addition, the image and point cloud data can be used to derive an accurate true ortho-photo mosaic, which will be obtained by simply projecting the colored point cloud on a specified datum (Ahmar et al., 1998; Habib et al., 2007). Different from the traditional true ortho-photos, which are produced by projecting the image data onto a horizontal datum, novel techniques have been developed to generate true orthophotos relative to any datum that will be specified by the users (Lari and Habib, 2015). These products provide a valuable source of information for different mapping, modelling and monitoring applications.

\subsection{Data processing}

To satisfy the needs of different mapping applications, the generated geo-referenced point cloud needs to undergo some processing to extract features of interest. The processing procedures should be developed to mitigate the impact of using low-cost geo-referencing and imaging sensors (mounted on low-cost UAV systems) on the quality of the extracted features. To date, different techniques have been introduced for the processing and information extraction from the unstructured point clouds (Filin and Pfeifer, 2006; Rabbani et al., 2006; Kim et al., 2007; Crosilla et al., 2009). The performance of the majority of these techniques is affected by internal characteristics of the generated point cloud (e.g., varying point density, noise level, surface orientation and shadow-induced color characteristics). However, these characteristics have not been effectively considered in previously-developed point cloud processing techniques. In recent years, a few research attempts have been made to introduce processing approaches adaptive to the varying characteristics of the utilized point cloud (AlDurgham and Habib, 2013; Lari and Habib, 2014). Qualitative analysis of the outcome from these approaches verified the impact of considering these characteristics for enhancing the performance of point cloud processing procedures. The integrated positional and spectral information within the extracted features (i.e., point cloud processing outcome) are then used to provide a preliminary interpretation of the source objects of those features (e.g., road surfaces, building rooftops and facades, parking lots, agricultural fields, power lines, light poles). The processed and simplified point cloud can finally be textured to provide a photo-realistic visualization and semantic information pertaining to individual scanned objects. Recently, region-based texturing techniques have been introduced that make the quality of final reconstructed surfaces (data processing products) independent of point density of the utilized point cloud (Lari and Habib, 2015). Figure 4 shows a 3D photorealistic model generated using a region-based texturing technique.

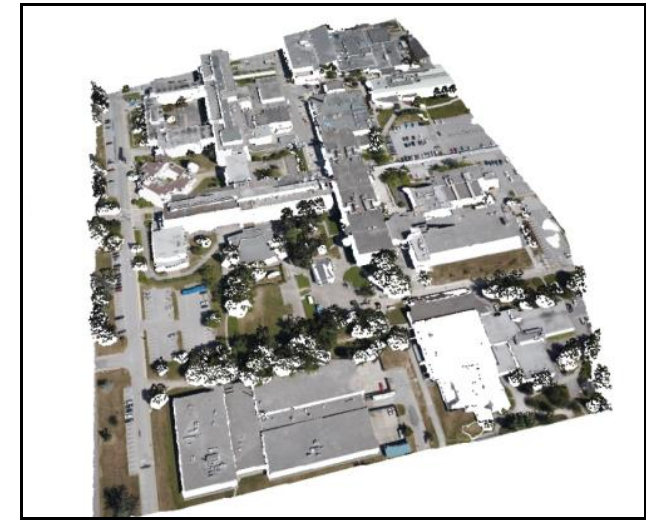

Figure 4: A photo-realistic 3D model generated using regionbased texturing technique

\subsection{Quality control of UAV-based mapping products}

Other than the previously-mentioned considerations for quality assurance of the collected data by low-cost UAV systems, quality control techniques are also required to evaluate the quality of intermediate as well as final mapping products. The most commonly used metrics for quality control of geospatial data processing outcome are correctness and completeness measures which are estimated based on confusion/error matrix (Heipke et al., 1997). Such quality control procedures can qualitatively evaluate the validity of the delivered product; however, they can't be applied in the absence of the reference data and do not investigate different issues affecting the processing outcome. In recent years, a few research attempts have been conducted to introduce new quality control techniques which address these issues and provide a quantitative evaluation of the semantic quality of the final product (i.e., extracted features) (Lari et al., 2014). Rather than traditional quality control procedures, which are implemented to either accept or reject the final product, these techniques propose solutions to remedy detected problems within the processing outcome. This paper provides a review of novel quality control procedures and their positive economic impact on the mapping process.

\section{CONCLUSION}

In the past few years, low-cost UAV systems have been evolved as a novel mobile mapping technology that can be utilized for different applications. In spite of its proven potential, the research related to UAV-based mapping is lacking in terms of what is needed to become an acceptable mapping tool. This technology should be applied while considering the posed challenges by using consumer-grade geo-referencing and imaging sensors to deliver accurate mapping products. This paper denoted these challenges and presented systematic, planning, and processing considerations for tackling them. These effective considerations will ensure an expedited achievement of a reliable UAV-based mapping procedure that is accepted by the data providers, end users, and regulatory organizations.

\section{ACKNOWLEDGEMENTS}

The authors would like to thank the Natural Sciences and Engineering Research Council of Canada Strategic Project Grants (NSERC SPG) for financial support of this research work. 


\section{REFERENCES}

Aber, J.S., Marzolff, I., Ries, J., 2010. Small-Format Aerial Photography: Principles, techniques and geoscience applications, 1 edition. Elsevier Science, Amsterdam.

Ahmar, F., Jansa, J., Ries, C., 1998. The generation of true orthophotos using a 3D building model in conjunction with a conventional DTM, in: ISPRS - International Archives of Photogrammetry, Remote Sensing and Spatial Information Science. Vol. 32(Part 4), pp. 16-22.

Al-Durgham, M., Habib, A., 2013. A framework for the registration and segmentation of heterogeneous LiDAR data. Photogramm. Eng. Remote Sens. 79 (2), 135-145.

Chiang, K.-W., Tsai, M.-L., Chu, C.-H., 2012. The development of an UAV borne direct georeferenced photogrammetric platform for ground control point free applications. Sensors 12 (7), 9161-9180.

Cramer, M., Stallmann, D., 2002. System calibration for direct georeferencing, in: ISPRS - International Archives of Photogrammetry, Remote Sensing and Spatial Information Sciences, Vol. 34 (Part 3/A). pp. 79-84.

Crosilla, F., Visintini, D., Sepic, F., 2009. Reliable automatic classification and segmentation of laser point clouds by statistical analysis of surface curvature values. Appl. Geomat. 1 (1-2), 17-30.

Eisenbeiss, H., 2011. The potential of Unmanned Aerial Vehicles for mapping. Presented at the Photogrammetric Week 2011, Stuttgart, Germany, pp. 135-145.

Eisenbeiss, H., 2009. UAV photogrammetry. ETH Zurich, Zurich, Switzerland.

El-Sheimy, N., 2005. An overview of mobile mapping systems, in: From Pharaohs to Geominformatics. Presented at the FIG Working Week 2005 and GSDI-8, Cairo, Egypt, pp. 124.

El-Sheimy, N., 1996. The development of VISAT : a mobile survey system for GIS applications. Department of Geomatics Engineering, University of Calgary, Calgary, Canada.

Everaerts, J., 2008. The use of unmanned aerial vehicles (UAVs) for remote sensing and mapping., in: ISPRS International Archives of the Photogrammetry, Remote Sensing and Spatial Information Sciences, Vol. 32, pp. 1187-1192.

Filin, S., Pfeifer, N., 2006. Segmentation of airborne laser scanning data using a slope adaptive neighborhood. ISPRS J. Photogramm. Remote Sens. 60 (2), 71-80.

Furukawa, Y., Ponce, J., 2010. Accurate, dense, and robust multiview stereopsis. IEEE Trans. Pattern Anal. Mach. Intell. 32 (8), 1362-1376.

Gehrke, S., Downey, M., Uebbing, R., Welter, J., LaRocque, W., 2012. A multi-sensor approach to semi-global matching. in: ISPRS - International Archives of the Photogrammetry, Remote Sensing and Spatial Information Sciences. Vol. 39 (Part B3), pp. 17-22.

Habib, A., Detchev, I., Kwak, E., 2014. Stability Analysis for a Multi-Camera Photogrammetric System. Sensors 14 (8), 15084-15112.

Habib, A., Kersting, A.P., Bang, K.-I., 2010. Stability Analysis for a Multi-Camera Photogrammetric System, in: Proceedings of Canadian Geomatics Conference 2010 and ISPRS COM I Symposium. Calgary, Canada.
Habib, A., Kersting, A.P., Bang, K.-I., Rau, J., 2011. A novel single-step procedure for the calibration of the mounting parameters of a multi-camera terrestrial mobile mapping system. Arch. Photogramm. Cartogr. Remote Sens. 22, 173-185.

Habib, A., Kim, E.M., Kim, C., 2007. New methodologies for true orthophoto generation. Photogramm. Eng. Remote Sens. 73 (1), 25-36.

Habib, A., Morgan, M., 2005. Stability analysis and geometric calibration of off-the-shelf digital cameras. Photogramm. Eng. Remote Sens. 71 (6), 733-741.

Habib, A., Pullivelli, A., Mitishita, E., Ghanma, M., Kim, E.M., 2006. Stability analysis of low-cost digital cameras for aerial mapping using different georeferencing techniques. Photogramm. Rec. 21 (113), 29-43.

He, F., Habib, A., 2014. Linear approach for initial recovery of the exterior orientation parameters of randomly captured images by low-cost mobile mapping systems, in: ISPRS International Archives of the Photogrammetry, Remote Sensing and Spatial Information Sciences. Vol. 40 (Part 1), pp. 149-154.

Heipke, C., Mayer, H., Wiedemann, C., Jamet, O., 1997. Evaluation of automatic road extraction, in: ISPRS International Archives of the Photogrammetry, Remote Sensing and Spatial Information Sciences, Vol. 32 (Part 32W3). pp. 47-56.

Hirschmuller, H., 2008. Stereo Processing by Semiglobal Matching and Mutual Information. IEEE Trans. Pattern Anal. Mach. Intell. 30 (2), 328-341.

Kersting, A.P., Habib, A., Bang, K.I., 2011. Mounting Parameters Calibration of GPS/INS-Assisted Photogrammetric Systems, in: 2011 International Workshop on Multi-Platform/Multi-Sensor Remote Sensing and Mapping (M2RSM), Xiamen, China, pp. 1-6.

Kim, C., Habib, A., Mrstik, P., 2007. New approach for planar patch segmentation using airborne and terrestrial laser data, in: Proceedings of ASPRS Annual Conference. Tampa, Florida, USA.

Kraus, K., 2007. Photogrammetry: geometry from images and laser scans. Walter de Gruyter.

Kwak, E., Habib, A., Tseng, Y.H., 2013. Comparative analysis of different approaches for multi-camera system calibration. J. Aeronaut. Astronaut. Aviat, 45 (Series A), 171-178.

Lari, Z., Al-Durgham, K., Habib, A., 2014. A novel quality control procedure for the evaluation of laser scanning data segmentation. in: ISPRS - International Archives of the Photogrammetry, Remote Sensing and Spatial Information Sciences. Vol. 40 (Part 1), pp. 207-210.

Lari, Z., Habib, A., 2015. A new approach for segmentationbased texturing of laser scanning data, in: ISPRS International Archives of the Photogrammetry, Remote Sensing and Spatial Information Sciences. Vol. 40 (Part 5W4), pp. 115-121.

Lari, Z., Habib, A., 2014. An adaptive approach for the segmentation and extraction of planar and linear/cylindrical features from laser scanning data. ISPRS J. Photogramm. Remote Sens. 93, 192-212.

Lari, Z., Habib, A., Mazaheri, M., Al-Durgham, K., 2014. Multi-camera System Calibration with Built-in Relative Orientation Constraints (Part 2) Automation, Implementation, and Experimental Results. J. Korean Soc. Surv. Geod. Photogramm. Cartogr. 32 (3), 205-216. 
Lichti, D., Habib, A., Detchev, I., 2009. An object-space simulation method for low-cost digital camera stability testing. Photogramm. Eng. Remote Sens. 75 (12), 14071414.

Lin, Y., Hyyppa, J., Jaakkola, A., 2011. Mini-UAV-borne LiDAR for fine-scale mapping. IEEE Geosci. Remote Sens. Lett. 8 (3), 426-430.

Nagai, M., Chen, T., Shibasaki, R., Kumagai, H., Ahmed, A., 2009. UAV-borne 3-D mapping system by multisensor integration. IEEE Trans. Geosci. Remote Sens. 47 (3), 701708.

Neitzel, F., Klonowski, J., 2012. Mobile 3D mapping with a low-cost UAV system. in: ISPRS - International Archives of the Photogrammetry, Remote Sensing and Spatial Information Sciences. Vol. 38 (Part 1-C22), pp. 39-44.

Nex, F., Remondino, F., 2014. UAV for 3D mapping applications: a review. Appl. Geomat. 6 (1), 1-15.

Pfeifer, N., Glira, P., Briese, C., 2012. Direct georeferencing with onboard navigation components of light weigh UAV platforms. in: ISPRS - International Archives of the Photogrammetry, Remote Sensing and Spatial Information Sciences. Vol. 39 (Part B7), pp. 487-492.

Pinto, L., Forlani, G., 2002. A single-step calibration procedure for IMU/GPS in aerial photogrammetry, in: ISPRS International Archives of the Photogrammetry, Remote Sensing and Spatial Information Sciences. Vol. 34 (Part 3), pp. 210-213.

Rabbani, T., van den Heuvel, F., Vosselman, G., 2006. Segmentation of point clouds using smoothness constraint, in: ISPRS - International Archives of the Photogrammetry, Remote Sensing and Spatial Information Sciences, Vol. 36 (Part5), pp. 248-253.

Rehak, M., Mabillard, R., Skaloud, J., 2013. A micro-UAV with the capability of direct georeferencing. in: ISPRS International Archives of the Photogrammetry, Remote Sensing and Spatial Information Sciences. Vol. 40 (Part 1/W2), pp. 317-323.

Rieke-Zapp, D., Tecklenburg, W., Peipe, J., Hastedt, H., Haig, C., 2009. Evaluation of the geometric stability and the accuracy potential of digital cameras - Comparing mechanical stabilisation versus parameterisation. ISPRS J. Photogramm. Remote Sens., 64 (3), 248-258.

Seitz, S.M., Curless, B., Diebel, J., Scharstein, D., Szeliski, R., 2006. A comparison and evaluation of multi-view stereo reconstruction algorithms, in: Proceedings of 2006 IEEE Computer Society Conference on Computer Vision and Pattern Recognition, New York, NY, USA, pp. 519-528.

Shortis, M.R., Beyer, H.A., 1997. Calibration stability of the Kodak DCS420 and 460 cameras. In: Proc. SPIE 3174, 94105.

Skaloud, J., 2007. Reliability of direct georeferencing - beyond the achilles heel of modern airborne mapping, in: Proceedings of Photogrammetric Week 2007. Wichmann, Heidelberg.

Tao, C.V., Li, J., 2007. Advances in Mobile Mapping Technology. CRC Press.

Wallace, L., Lucieer, A., Watson, C., Turner, D., 2012. Development of a UAV-LiDAR System with Application to forest inventory. Remote Sens. 4 (6), 1519-1543.

Zhou, G., Yang, J., Li, X., Yang, X., 2012. Advances of flash LiDAR development onboard UAV, in: ISPRS International Archives of the Photogrammetry, Remote
Sensing and Spatial Information Sciences. Vol. 39 (Part B3), pp. 193-198.

Zhu, Q., Zhang, Y., Wu, B., Zhang, Y., 2010. Multiple closerange image matching based on a self-adaptive triangle constraint. Photogramm. Rec. 25 (132), 437-453. 